\title{
A Two-Level Additive Schwarz Preconditioning Algorithm for the Weak Galerkin Method for the Second-Order Elliptic Equation
}

\author{
Fangfang Qin, Min Zha, and Feng Wang \\ Jiangsu Key Laboratory for NSLSCS, School of Mathematical Sciences, Nanjing Normal University, Nanjing 210023, China \\ Correspondence should be addressed to Feng Wang; fengwang@live.cn
}

Received 16 December 2015; Accepted 9 March 2016

Academic Editor: Yann Favennec

Copyright (C) 2016 Fangfang Qin et al. This is an open access article distributed under the Creative Commons Attribution License, which permits unrestricted use, distribution, and reproduction in any medium, provided the original work is properly cited.

This paper proposes a two-level additive Schwarz preconditioning algorithm for the weak Galerkin approximation of the secondorder elliptic equation. In the algorithm, a $P_{1}$ conforming finite element space is defined on the coarse mesh, and a stable intergrid transfer operator is proposed to exchange the information between the spaces on the coarse mesh and the fine mesh. With the framework of the Schwarz method, it is proved that the condition number of the preconditioned system only depends on the rate of the coarse mesh size and the overlapping size. Some numerical experiments are carried out to verify the theoretical results.

\section{Introduction}

The weak Galerkin (WG) finite element method (FEM) is a new efficient numerical method for solving partial differential equations. It was first introduced for the second-order elliptic problem in [1]. The central idea of the WG method is using weak derivatives in place of strong derivatives that define the weak formulation for the underlying partial differential equations. Due to the weak derivative, the degrees of freedom can be discontinuous from the element interior to the element boundary, which makes the weak Galerkin method have more choices of the finite elements than the standard FEMs. The WG FEM also has some other properties, such as high-order accuracy and multiphysics capability. The idea of the weak derivative has been generalized to some other problems, such as the biharmonic equation [2] and the twophase subsurface flow problems [3]. Chen et al. introduced a posteriori error estimates for the WG method for the secondorder elliptic problems in [4].

Although many works focus on developing WG methods for different problems, there are few works on efficiently solving the discretized systems, of which the condition number is $O\left(h^{-2}\right)$, where $h$ denotes the mesh size. Recently,
$\mathrm{Li}$ and Xie [5] proposed a multigrid algorithm for the WG method using $P_{1}$ conforming element on the coarser meshes. In [6], Chen et al. also constructed a fast auxiliary space multigrid preconditioner for the WG method, as well as a corresponding reduced system involving only the degrees of freedom on edges/faces. It is proved that the condition number of the preconditioned system is independent of the mesh size.

Due to its high parallelizability and scalability, the domain decomposition method is one of the most efficient algorithms for the numerical solutions of partial differential equations (see, e.g., [7]). In this paper, we study an overlapping domain decomposition algorithm and propose a two-level additive Schwarz preconditioner for the WG method for the secondorder elliptic equation. The preconditioner is consisted of overlapping local subproblems on a fine mesh and a global subproblem on a coarse mesh. The coarse subproblem is defined by the $P_{1}$ conforming element method, and an intergrid transfer operator, which has stability and approximability, is introduced to transmit information between the coarse mesh and the fine mesh. Under the Schwarz framework, we prove that the condition number of the preconditioned system is independent of the fine mesh size $h$ and is of $O((1+$ 
$H / \delta)^{2}$ ), where $H$ denotes the coarse mesh size and $\delta$ stands for the overlapping size.

The rest of this paper is organized as follows. In Section 2, we recall the WG method and give some notations. In Section 3, we propose an intergrid transfer operator and a two-level additive Schwarz preconditioner. Moreover, we prove the stability and approximation of the intergrid transfer operator. Then we estimate the upper bound of the maximum eigenvalue and the lower bound of minimum eigenvalue, respectively. Section 4 is devoted to numerical experiments, which are carried out to confirm our theoretical results.

\section{The Weak Galerkin Method for the Second-Order Elliptic Equation}

For the sake of simplicity, we consider the model problem as follows:

$$
\begin{aligned}
-\nabla \cdot(\mathbb{A} \nabla u) & =f, \quad \text { in } \Omega, \\
u=0, & \text { on } \partial \Omega,
\end{aligned}
$$

where $\Omega \subset \mathbb{R}^{2}$ is bounded polygonal domain and $f \in L^{2}(\Omega)$. Assume that the matrix $\mathbb{A}$ is symmetric positive definite (SPD); namely, there exist positive constants $\alpha, \beta$ such that

$$
\alpha \xi^{T} \xi \leq \xi^{T} \mathbb{A}(x) \xi \leq \beta \xi^{T} \xi, \quad \forall \xi \in \mathbb{R}^{2}, x \in \Omega .
$$

The variational formulations of (1) is to find $u \in H_{0}^{1}(\Omega)$ such that

$$
a(u, v)=(f, v) \quad \forall v \in H_{0}^{1}(\Omega),
$$

where $a(u, v)=(\mathbb{A} \nabla u, \nabla v)$. It follows from Lax-Milgram's theorem that problem (3) has a unique solution.

Let $\mathscr{T}_{h}$ be a quasiuniform triangulation of the domain $\Omega$ with the mesh size $h$. On each $T \in \mathscr{T}_{h}$, the space $W(T)$ denotes the collections of weak functions, each of which is consisted of an interior part and a boundary part; that is,

$$
W(T):=\left\{v=\left\{v_{0}, v_{b}\right\}: v_{0} \in L^{2}(T), v_{b} \in H^{1 / 2}(\partial T)\right\} .
$$

For any weak function $v \in W(T)$, the weak gradient of $v$, denoted by $\nabla_{w} v \in H(\operatorname{div}, T)$, satisfies

$$
\begin{aligned}
\int_{T} \nabla_{w} v \cdot \mathbf{q} d x=-\int_{T} v_{0} \nabla \cdot \mathbf{q} d x+\int_{\partial T}{ }_{b} \mathbf{q} \cdot \mathbf{n} d s & \\
& \forall \mathbf{q} \in H(\operatorname{div}, T),
\end{aligned}
$$

where $\mathbf{n}$ is the unit outer normal of $\partial T$. It is easy to see that $\nabla_{w}$ is the classical gradient if it actions on a function $u \in H^{1}(T)$.

For each element $T \in \mathscr{T}_{h}$, we denote by $T_{0}$ and $\partial T$ the interior and the boundary of $T$. The notation $\mathscr{F}_{T}$ stands for the set of edges of $T$. We also use $\mathscr{F}_{h}$ and $\mathscr{F}_{h}^{\partial}$ to denote the edges of $\mathscr{T}_{h}$ in $\Omega$ and on $\partial \Omega$. Then the discrete WG space is defined as

$$
\begin{aligned}
W_{h} & :=\left\{v:\left.v\right|_{T_{0}} \in P_{j}\left(T_{0}\right) \forall T \in \mathscr{T}_{h},\left.v\right|_{F} \in P_{l}(F) \forall F\right. \\
& \left.\in \mathscr{F}_{h},\left.v\right|_{F}=0 \forall F \in \mathscr{F}_{h}^{\partial}\right\},
\end{aligned}
$$

where $l=j$ or $l=j+1$ and $P_{j}\left(T_{0}\right)$ denotes the set of polynomials on $T_{0}$ with degree no more than $j \geq 0$. To define a discrete weak gradient, we use $G_{j}(T)$ to denote the RT element space $\left[P_{j}(T)\right]^{2}+\widehat{P}_{j}(T) x$ if $l=j$ and the BDM element space $\left[P_{j+1}(T)\right]^{2}$ if $l=j+1$, where $\widehat{P_{j}}(T)$ is the homogeneous polynomial with the degree $j$.

For each $v \in W_{h}$, the discrete weak gradient $\nabla_{w} v \in G_{j}(T)$ is defined as

$$
\begin{array}{r}
\int_{T} \nabla_{w} v \cdot \mathbf{q} d x=-\int_{T} v_{0}(\nabla \cdot \mathbf{q}) d x+\int_{\partial T} v_{b}(\mathbf{q} \cdot \mathbf{n}) d s \\
\forall \mathbf{q} \in G_{j}(T),
\end{array}
$$

where $v_{0}$ and $v_{b}$ denote the values of $v$ in the interior and on the boundary of $T$. Then the discrete problem for (3) is to seek $u_{h}=\left\{u_{0}, u_{b}\right\} \in W_{h}$ such that

$$
a_{h}\left(u_{h}, v_{h}\right)=\left(f, v_{0}\right), \quad \forall v_{h}=\left\{v_{0}, v_{b}\right\} \in W_{h},
$$

where $a_{h}\left(u_{h}, v_{h}\right):=\left(\mathbb{A} \nabla_{w} u_{h}, \nabla_{w} v_{h}\right)$

For any $v_{h}=\left\{v_{0}, v_{b}\right\}$ and $u_{h}=\left\{u_{0}, u_{b}\right\}$ in $W_{h}$, we use $\left(\left(u_{h}, v_{h}\right)\right)$ to indicate a special inner product defined as

$$
\left(\left(v_{h}, u_{h}\right)\right):=\sum_{T \in \mathscr{T}_{h}}\left[\left(v_{0}, u_{0}\right)_{T}+h\left(v_{0}-v_{b}, u_{0}-u_{b}\right)_{\partial T}\right]
$$

where $(\cdot, \cdot)_{T}$ and $(\cdot, \cdot)_{\partial T}$ denote the $L^{2}$ inner product on $T$ and $\partial T$. Accordingly, a norm and seminorm are introduced for any $v_{h}=\left\{v_{0}, v_{b}\right\} \in W_{h}$ by

$$
\begin{aligned}
\left\|v_{h}\right\|_{0, h} & :=\left(\sum_{T \in \mathscr{T}_{h}}\left\|v_{h}\right\|_{0, h, T}^{2}\right)^{1 / 2}, \\
\left|v_{h}\right|_{1, h} & :=\left(\sum_{T \in \mathscr{T}_{h}}\left|v_{h}\right|_{1, h, T}^{2}\right)^{1 / 2},
\end{aligned}
$$

where $\left\|v_{h}\right\|_{0, h, T}:=\left(\left\|v_{0}\right\|_{0, T}^{2}+h\left\|v_{0}-v_{b}\right\|_{\partial T}^{2}\right)^{1 / 2}$ and $\left|v_{h}\right|_{1, h, T}:=$ $\left(\left|v_{0}\right|_{1, T}^{2}+h^{-1}\left\|v_{0}-v_{b}\right\|_{\partial T}^{2}\right)^{1 / 2}$.

Let $A_{h}: W_{h} \rightarrow W_{h}$ be an operator

$$
\left(\left(A_{h} u_{h}, v_{h}\right)\right)=\left(\mathbb{A} \nabla_{w} u_{h}, \nabla_{w} v_{h}\right), \quad \forall u_{h}, v_{h} \in W_{h}
$$

It can be proved that $A_{h}$ is symmetric and positive definite and the condition number is of $O\left(h^{-2}\right)$ (see, e.g., [8]), which brings difficulty to solve the discrete problem when the mesh size is small. In the next section, we will present a preconditioner to overcome this difficulty.

\section{The Overlapping Domain Decomposition Method}

3.1. A Two-Level Additive Schwarz Preconditioner. To introduce our preconditioner, we first divide the domain $\Omega$ by $J$ overlapping subdomains $\Omega_{1}, \Omega_{2}, \ldots, \Omega_{J}$ such that each point in $\Omega$ belongs to no more than $N_{C}$ subdomains. We assume that the boundary of each subdomain does not cut through any elements in the triangulation $\mathscr{T}_{h}$, and there 
exist nonnegative $C^{\infty}$ functions $\theta_{1}, \theta_{2}, \ldots, \theta_{J}$ satisfying the following properties: (1) $\theta_{j}=0$ in $\Omega \backslash \Omega_{j}$; (2) $\sum_{j=1}^{J} \theta_{j}=1$; (3) there exists a positive constant $\delta$, such that $\left|\nabla \theta_{j}\right| \leq C / \delta$, where $C$ is a constant independent of $\delta, h$, and $J$.

On each subdomain $\Omega_{j}$, the notation $\mathscr{T}_{h, j}$ stands for the triangulation inherited from $\mathscr{T}_{h}$. The corresponding weak Galerkin subspace on $\mathscr{T}_{h, j}$ is defined as

$$
V_{j}=\left\{v \in W_{h}:\left.v\right|_{\Omega \backslash \Omega_{j}}=0\right\} .
$$

We introduce an operator $A_{j}: V_{j} \rightarrow V_{j}$ by

$$
\left(\left(A_{j} u_{j}, v_{j}\right)\right)=\left(A \nabla_{w} u_{j}, \nabla_{w} v_{j}\right), \quad \forall u_{j}, v_{j} \in V_{j} .
$$

It is easy to see that $A_{j}$ is symmetric and positive definite. Since $V_{j}$ is a subset of $W_{h}$, we use $I_{j}$ to denote a natural injection from $V_{j}$ to $W_{h}$.

To define a coarse subproblem, we define a coarse triangulation $\mathscr{T}_{H}$ with mesh size $H$ such that each element in $\mathscr{T}_{h}$ is a subdivision of the one in $\mathscr{T}_{H}$. The notation $V_{H} \subset H_{0}^{1}(\Omega)$ stands for $P_{1}$ conforming finite element space associated with $\mathscr{T}_{H}$. We also introduce an operator $A_{H}: V_{H} \rightarrow V_{H}$ satisfying

$$
\left(A_{H} u_{H}, v_{H}\right)=\left(\AA \nabla u_{H}, \nabla v_{H}\right), \quad \forall u_{H}, v_{H} \in V_{H} .
$$

Note that the coarse space $V_{H}$ is a subspace of $W_{h}$ if the degree of the piecewise polynomials in $W_{h}$ is greater than 0 , and we can choose a natural injection as the intergird transfer operator $I_{H}$ from $V_{H}$ to $W_{h}$. For the piecewise constant space case, the intergrid transfer operator $I_{H}: V_{H} \rightarrow W_{h}$ is defined as

$$
I_{H} v_{H}=\left\{I_{H}^{0} v_{H}, I_{H}^{b} v_{H}\right\}, \quad \forall v_{H} \in V_{H},
$$

where $I_{H}^{0}$ and $I_{H}^{b}$ satisfy

$$
\begin{aligned}
& \left.\left(I_{H}^{0} v_{H}\right)\right|_{T}=\frac{1}{|T|} \int_{T} v_{H} d x, \\
& \left.\left(I_{H}^{b} v_{H}\right)\right|_{F}=\frac{1}{|F|} \int_{F} v_{H} d s,
\end{aligned}
$$

for any $T \in \mathscr{T}_{h}$ and $F \in \mathscr{F}_{h}$. We also need the transpose of the intergrid transfer operators $I_{j}^{t}$ from $W_{h}$ to the subspace $V_{j}$ for $j=H, 1,2, \ldots, J$, defined as

$$
\left(\left(I_{j}^{t} u_{h}, v_{j}\right)\right)=\left(\left(u_{h}, I_{j} v_{j}\right)\right), \quad \forall u_{h} \in W_{h}, v_{j} \in V_{j} .
$$

Then our two-level additive Schwarz preconditioner $B_{h}$ is stated as

$$
B_{h}=I_{H} A_{H}^{-1} I_{H}^{t}+\sum_{j=1}^{J} I_{j} A_{j}^{-1} I_{j}^{t}
$$

3.2. Analysis. Our analysis is based on the standard Schwarz framework (see, e.g., [7, 9]).
Lemma 1 ([9, Theorem 7.1.20]). The eigenvalues of $B_{h} A_{h}$ are positive, and one has the following characterizations of the maximum and minimum eigenvalues:

$$
\begin{aligned}
& \lambda_{\max }\left(B_{h} A_{h}\right) \\
& =\max _{v \in W_{h}, v \neq 0} \frac{\left(\left(A_{h} v, v\right)\right)}{\min _{v=\sum_{j, v_{j} \in V_{j}} I_{j} v_{j}} \sum_{j}\left(\left(A_{j} v_{j}, v_{j}\right)\right)}, \\
& \lambda_{\min }\left(B_{h} A_{h}\right) \\
& =\min _{v \in W_{h}, v \neq 0} \frac{\left(\left(A_{h} v, v\right)\right)}{\min _{v=\sum_{j, v_{j} \in V_{j}} I_{j} v_{j}} \sum_{j}\left(\left(A_{j} v_{j}, v_{j}\right)\right)},
\end{aligned}
$$

where the sum is taken over $j=H, 1, \ldots, J$.

Lemma 2. For the intergrid transfer operator $I_{H}$, it holds for any $v_{H} \in V_{H}$ that

$$
\left\|v_{H}-I_{H} v_{H}\right\|_{0, h}^{2}+h^{2}\left|I_{H} v_{H}\right|_{1, h}^{2} \leqslant h^{2}\left|v_{H}\right|_{1}^{2} .
$$

Proof. We only need consider the case that the functions in $W_{h}$ are the constants in the interior and on the boundary of each element.

It follows from the triangle inequality, the trace inequality, and the Poincaré-Friedrichs inequality that

$$
\begin{aligned}
& \left|I_{H} v_{H}\right|_{1, h}^{2}=\sum_{T \in \mathscr{T}_{h}} h^{-1}\left\|I_{H}^{0} v_{H}-I_{H}^{b} v_{H}\right\|_{0, \partial T}^{2} \\
& \quad \leq \sum_{T \in \mathscr{T}_{h}} h^{-1}\left(\left\|I_{H}^{0} v_{H}-v_{H}\right\|_{0, \partial T}^{2}+\left\|I_{H}^{b} v_{H}-v_{H}\right\|_{0, \partial T}^{2}\right) \\
& \quad=\left|v_{H}\right|_{1}^{2} .
\end{aligned}
$$

Similarly, for the lower-order term, we have

$$
\begin{aligned}
& \left\|v_{H}-I_{H} v_{H}\right\|_{0, h}^{2} \\
& =\sum_{T \in \mathscr{T}_{h}}\left(\left\|v_{H}-I_{H}^{0} v_{H}\right\|_{0, T}^{2}+h\left\|I_{H}^{0} v_{H}-I_{H}^{b} v_{H}\right\|_{0, \partial T}^{2}\right) \\
& \quad \leq h^{2}\left|v_{H}\right|_{1}^{2},
\end{aligned}
$$

which completes the proof.

Lemma 3. There exists an operator $Q^{H}$ from $W_{h}$ to $V_{H}$ such that for any $v_{h}$ in $W_{h}$ it holds that

$$
\left\|v_{h}-Q^{H} v_{h}\right\|_{0, h}^{2}+H^{2}\left|Q^{H} v_{h}\right|_{1}^{2} \leqslant H^{2}\left|v_{h}\right|_{1, h}^{2} .
$$

Proof. Let $V_{h}$ be the $P_{1}$ conforming finite element space defined on $\mathscr{T}_{h}$. Then, for any $v_{h}$ in $W_{h}$, we can construct a $\widetilde{v}_{h} \in V_{h}$ satisfying ([6, Lemma 3.5])

$$
\left\|v_{h}-\widetilde{v}_{h}\right\|_{0, h}+h\left|\widetilde{v}_{h}\right|_{1, h} \leqslant h\left|v_{h}\right|_{1, h} .
$$


4

Mathematical Problems in Engineering

Define $Q^{H} v_{h}=Q_{H} \widetilde{v}_{h}$, where $Q_{H}$ is the $L^{2}$ projection operator from $V_{h}$ to $V_{H}$. Using the standard properties of $Q_{H}$ (see, e.g., [7]) and the inequality (24), we have

$$
\begin{aligned}
\left|Q^{H} v_{h}\right|_{1}^{2} & =\left|Q_{H} \widetilde{v}_{h}\right|_{1}^{2} \lesssim\left|\widetilde{v}_{h}\right|_{1}^{2} \lesssim\left|v_{h}\right|_{1, h}^{2}, \\
\left\|v_{h}-Q^{H} v_{h}\right\|_{0, h}^{2} & =\left\|v_{h}-Q_{H} \widetilde{v}_{h}\right\|_{0, h}^{2} \\
& \leq\left\|v_{h}-\widetilde{v}_{h}\right\|_{0, h}^{2}+\left\|Q_{H} \widetilde{v}_{h}-\widetilde{v}_{h}\right\|_{0, h}^{2} \\
& \lesssim h^{2}\left|v_{h}\right|_{1, h}^{2}+H^{2}\left|\widetilde{v}_{h}\right|_{1}^{2} \lesssim H^{2}\left|v_{h}\right|_{1, h}^{2} .
\end{aligned}
$$

The proof is completed.

Lemma 4. Given any $v_{h} \in W_{h}$, there exists a decomposition

$$
v_{h}=I_{H} v_{H}+\sum_{j=1}^{J} I_{j} v_{j},
$$

where $v_{H} \in V_{H}$ and $v_{j} \in V_{j}$, such that

$$
\begin{gathered}
\left(A_{H} v_{H}, v_{H}\right)+\sum_{j=1}^{J}\left(\left(A_{j} v_{j}, v_{j}\right)\right) \\
\lesssim\left(1+\frac{H}{\delta}\right)^{2}\left(\left(A_{h} v_{h}, v_{h}\right)\right) .
\end{gathered}
$$

Proof. Let $v_{H}=Q^{H} v, w=v_{h}-I_{H} v_{H}$, and $v_{j}=Q_{h}\left(\theta_{j} w\right)=$ $\left\{Q_{h}^{0}\left(\theta_{j} w_{0}\right), Q_{h}^{b}\left(\theta_{j} w_{b}\right)\right\}$, where $Q_{h}^{0}$ and $Q_{h}^{b}$ are the piecewise $L^{2}$ projection to the interior polynomial space $P_{j}\left(T_{0}\right)$ and the edge polynomial space $P_{l}(F)$, respectively. It is easy to check that

$$
\begin{aligned}
I_{H} v_{H}+\sum_{j=1}^{J} v_{j} & =I_{H} v_{H}+\sum_{j=1}^{J} Q_{h}\left(\theta_{j} w\right) \\
& =I_{H} v_{H}+Q_{h}\left(\sum_{j=1}^{J}\left(\theta_{j} w\right)\right) \\
& =I_{H} v_{H}+Q_{h} w=v_{h} .
\end{aligned}
$$

It follows from Lemma 3 that

$$
\left(A_{H} v_{H}, v_{H}\right) \lesssim\left|v_{H}\right|_{1}^{2} \lesssim\left|v_{h}\right|_{1, h}^{2} \lesssim\left(\left(A_{h} v_{h}, v_{h}\right)\right) \text {. }
$$

On the other hand, we have for $v_{j}$ that

$$
\begin{aligned}
& \left(\left(A_{j} v_{j}, v_{j}\right)\right) \lesssim \sum_{T \in \mathscr{T}_{h, j}}\left|v_{j}\right|_{1, h, T}^{2}=\sum_{T \in \mathscr{T}_{h, j}}\left(\left|Q_{h}^{0}\left(\theta_{j} w_{0}\right)\right|_{1, T}^{2}\right. \\
& \left.+h^{-1}\left\|Q_{h}^{0}\left(\theta_{j} w_{0}\right)-Q_{h}^{b}\left(\theta_{j} w_{b}\right)\right\|_{0, \partial T}^{2}\right) .
\end{aligned}
$$

By the inverse inequality, the stability of $L^{2}$ projection, the Poincare inequality, and the scaling argument, we deduce that

$$
\begin{aligned}
\left|Q_{h}^{0}\left(\theta_{j} w_{0}\right)\right|_{1, T}^{2} & =\inf _{c \in \mathbb{R}}\left|Q_{h}^{0}\left(\theta_{j} w_{0}-c\right)\right|_{1, T}^{2} \\
& \lesssim \inf _{c \in \mathbb{R}} h^{-1}\left\|Q_{h}^{0}\left(\theta_{j} w_{0}-c\right)\right\|_{0, T}^{2} \\
& \leq \inf _{c \in \mathbb{R}} h^{-1}\left\|\theta_{j} w_{0}-c\right\|_{0, T}^{2} \lesssim\left|\theta_{j} w_{0}\right|_{1, T}^{2},
\end{aligned}
$$

which, together with the triangle inequality and the assumpion of $\theta_{j}$, yields

$$
\begin{aligned}
& \left(\left(A_{j} v_{j}, v_{j}\right)\right) \lesssim \sum_{T \in \mathscr{T}_{h, j}}\left(\left|\theta_{j} w_{0}\right|_{1, T}^{2}\right. \\
& \left.+h^{-1}\left\|Q_{h}^{0}\left(\theta_{j} w_{0}\right)-Q_{h}^{b}\left(\theta_{j} w_{b}\right)\right\|_{0, \partial T}^{2}\right) \\
& \quad \lesssim \sum_{T \in \mathscr{T}_{h, j}}\left(\int_{T}\left|\left(\nabla \theta_{j} \cdot w_{0}+\nabla w_{0} \cdot \theta_{j}\right)\right|^{2} d x\right. \\
& \left.+h^{-1}\left\|Q_{h}^{0}\left(\theta_{j} w_{0}\right)-Q_{h}^{b}\left(\theta_{j} w_{b}\right)\right\|_{0, \partial T}^{2}\right) \\
& \quad \leq \sum_{T \in \mathscr{T}_{h, j}}\left(2\left\|\nabla \theta_{j} \cdot w_{0}\right\|_{0, T}^{2}+2\left\|\nabla w_{0} \cdot \theta_{j}\right\|_{0, T}^{2}\right. \\
& \left.+h^{-1}\left\|Q_{h}^{0}\left(\theta_{j} w_{0}\right)-Q_{h}^{b}\left(\theta_{j} w_{b}\right)\right\|_{0, \partial T}^{2}\right) \\
& \quad \lesssim \sum_{T \in \mathscr{T}_{h, j}}\left(\frac{1}{\delta^{2}}\left\|w_{0}\right\|_{0, T}^{2}+\left|w_{0}\right|_{1, T}^{2}\right. \\
& \left.+h^{-1}\left\|Q_{h}^{0}\left(\theta_{j} w_{0}\right)-Q_{h}^{b}\left(\theta_{j} w_{b}\right)\right\|_{0, \partial T}^{2}\right) .
\end{aligned}
$$

For the last term, the triangle inequality gives

$$
\begin{aligned}
& \left\|Q_{h}^{0}\left(\theta_{j} w_{0}\right)-Q_{h}^{b}\left(\theta_{j} w_{b}\right)\right\|_{0, \partial T}^{2} \\
& \lesssim\left\|Q_{h}^{0}\left(\theta_{j} w_{0}\right)-\theta_{j} w_{0}\right\|_{0, \partial T}^{2} \\
& \quad+\left\|\theta_{j} w_{0}-Q_{h}^{b}\left(\theta_{j} w_{0}\right)\right\|_{0, \partial T}^{2} \\
& \quad+\left\|Q_{h}^{b}\left(\theta_{j} w_{0}\right)-Q_{h}^{b}\left(\theta_{j} w_{b}\right)\right\|_{0, \partial T}^{2}=\mathrm{I}+\mathrm{II}+\mathrm{III} .
\end{aligned}
$$

We estimate I, II, and III, respectively, as follows.

From the trace theorem, the scaling argument, and the stability and approximation of $Q_{h}^{0}$, we have

$$
\begin{aligned}
I= & \left\|Q_{h}^{0}\left(\theta_{j} w_{0}\right)-\theta_{j} w_{0}\right\|_{0, \partial T}^{2} \\
\lesssim & h^{-1}\left\|Q_{h}^{0}\left(\theta_{j} w_{0}\right)-\theta_{j} w_{0}\right\|_{0, T}^{2} \\
& +h\left|Q_{h}^{0}\left(\theta_{j} w_{0}\right)-\theta_{j} w_{0}\right|_{1, T}^{2} \leq h\left|\theta_{j} w_{0}\right|_{1, T}^{2} \\
& \leqslant \frac{h}{\delta^{2}}\left\|w_{0}\right\|_{0, T}^{2}+h\left|w_{0}\right|_{1, T}^{2} .
\end{aligned}
$$


Denote $\overline{\theta_{j} w_{0}}=(1 /|T|) \int_{T} \theta_{j} w_{0} d x$. The trace theorem, the $L^{2}$ stability of $Q_{h}^{b}$, and the Poincaré inequality imply that

$$
\begin{aligned}
\mathrm{II} & =\left\|\theta_{j} w_{0}-Q_{h}^{b}\left(\theta_{j} w_{0}\right)\right\|_{0, \partial T}^{2} \\
& \leq\left\|\theta_{j} w_{0}-\overline{\theta_{j} w_{0}}\right\|_{0, \partial T}^{2}+\left\|Q_{h}^{b}\left(\theta_{j} w_{0}-\overline{\theta_{j} w_{0}}\right)\right\|_{0, \partial T}^{2} \\
& \leq\left\|\theta_{j} w_{0}-\overline{\theta_{j} w_{0}}\right\|_{0, \partial T}^{2} \lesssim h\left|\theta_{j} w_{0}\right|_{1, T}^{2} \\
& \leq \frac{h}{\delta^{2}}\left\|w_{0}\right\|_{0, T}^{2}+h\left|w_{0}\right|_{1, T}^{2} .
\end{aligned}
$$

Simiarily, we obtain

$$
\begin{aligned}
\mathrm{III} & =\left\|Q_{h}^{b}\left(\theta_{j} w_{0}\right)-Q_{h}^{b}\left(\theta_{j} w_{b}\right)\right\|_{0, \partial T}^{2} \\
& \lesssim\left\|\theta_{j} w_{0}-\theta_{j} w_{b}\right\|_{0, \partial T}^{2} \leq\left\|w_{0}-w_{b}\right\|_{0, \partial T}^{2} .
\end{aligned}
$$

Combining the above five inequalities, we find

$$
\begin{aligned}
& \left(\left(A_{j} v_{j}, v_{j}\right)\right) \\
& \lesssim \sum_{T \in \mathscr{T}_{h, j}}\left[\frac{1}{\delta^{2}}\left\|w_{0}\right\|_{0, T}^{2}+\left|w_{0}\right|_{1, T}^{2}+h^{-1}\left\|w_{0}-w_{b}\right\|_{0, \partial T}^{2}\right],
\end{aligned}
$$

which leads to

$$
\begin{aligned}
& \sum_{j=1}^{J}\left(\left(A_{j} v_{j}, v_{j}\right)\right) \lesssim \sum_{j=1}^{J} \sum_{T \in \mathscr{T}_{h, j}}\left[\frac{1}{\delta^{2}}\left\|w_{0}\right\|_{0, T}^{2}+\left|w_{0}\right|_{1, T}^{2}\right. \\
& \left.+h^{-1}\left\|w_{0}-w_{b}\right\|_{0, \partial T}^{2}\right] \lesssim\left(\frac{1}{\delta^{2}}\left\|v_{h}-I_{H} v_{H}\right\|_{0, h}^{2}+\mid v_{h}\right. \\
& \left.-\left.I_{H} v_{H}\right|_{1, h} ^{2}\right) \leq\left(\frac{1}{\delta^{2}}\left\|v_{h}-v_{H}\right\|_{0, h}^{2}+\frac{1}{\delta^{2}} \| v_{H}\right. \\
& \left.-I_{H} v_{H} \|_{0, h}^{2}+\left|v_{h}-I_{H} v_{H}\right|_{1, h}^{2}\right) \lesssim\left(1+\frac{H^{2}}{\delta^{2}}\right)\left|v_{h}\right|_{1, h}^{2} \\
& \quad \leq\left(1+\frac{H}{\delta}\right)^{2}\left(\left(A_{h} v_{h}, v_{h}\right)\right) .
\end{aligned}
$$

Using (27) and (38), we achieve

$$
\begin{gathered}
\left(A_{H} v_{H}, v_{H}\right)+\sum_{j=1}^{J}\left(\left(A_{j} v_{j}, v_{j}\right)\right) \\
\lesssim\left(1+\frac{H}{\delta}\right)^{2}\left(\left(A_{h} v_{h}, v_{h}\right)\right) .
\end{gathered}
$$

This ends the proof.

Lemma 5. Let $v_{H} \in V_{H}$ and $v_{j} \in V_{j}, 1 \leq j \leq J$. For any $v_{h}=I_{H} v_{H}+\sum_{j=1}^{J} v_{j}$, it is true that

$$
\left(\left(A_{h} v_{h}, v_{h}\right)\right) \lesssim\left(A_{H} v_{H}, v_{H}\right)+\sum_{j=1}^{J}\left(\left(A_{j} v_{j}, v_{j}\right)\right) .
$$

TABLE 1: The iteration numbers of the PCG and CG.

\begin{tabular}{lccccc}
\hline$h$ & $1 / 8$ & $1 / 16$ & $1 / 32$ & $1 / 64$ & $1 / 128$ \\
\hline PCG $(H / \delta=1)$ & 10 & 11 & 11 & 11 & 12 \\
\hline PCG $(H / \delta=2)$ & 14 & 15 & 15 & 16 & 16 \\
\hline CG & 60 & 119 & 231 & 436 & 864 \\
\hline
\end{tabular}

Proof. The inequality can be obtained directly by using the triangle inequality and the assumption on the finite cover of subdomains.

As an immediate consequence, we have the following theorem.

Theorem 6. There exists a positive constant $C$, independent of $H, h, \delta$, and $J$, such that

$$
\kappa\left(B_{h} A_{h}\right)=\frac{\lambda_{\text {max }}\left(B_{h} A_{h}\right)}{\lambda_{\min }\left(B_{h} A_{h}\right)} \leq C\left(1+\frac{H}{\delta}\right)^{2} .
$$

Remark 7. According the theorem, the two-level additive preconditioner is optimal if $H / \delta$ is bounded above by a constant. In particular, when $\delta=O(H)$, we have

$$
\kappa\left(B_{h} A_{h}\right) \leqslant C \text {. }
$$

\section{Numerical Experiments}

In this section, we give some numerical results to demonstrate the efficiency of our preconditioner. For convenience, we consider a simple two-dimensional Poisson equation with homogeneous boundary as follows:

$$
\begin{array}{rr}
-\Delta u=f & \text { in } \Omega, \\
u=0 & \text { on } \partial \Omega .
\end{array}
$$

We choose $f$ to satisfy the exact solution $u\left(x_{1}, x_{2}\right)=$ $x_{1}\left(1-x_{1}\right) x_{2}\left(1-x_{2}\right)$ on $\Omega=[0,1] \times[0,1]$. Let $\mathscr{T}_{H}$ be a uniform triangulation with the mesh size $H=1 / 4$, and let $\mathscr{T}_{h}$ be the refinement of $\mathscr{T}_{H}$. All the tests are stopped when the relative error is less than $10^{-6}$.

In Table 1, we list the iteration numbers of the preconditioning conjugate gradient (PCG) method and the conjugate gradient (CG) method with different meshes. From the second and third rows of the table, we see that the iteration numbers of the PCG method are almost the same if the overlapping factors $H / \delta$ are fixed. If the overlap becomes small, that is, the rate $H / \delta$ is increasing, one needs a little more steps to achieve the tolerance. This indicates that the condition number of the preconditioned system is almost a constant which is independent of the mesh size $h$ and only depends on the rate of the coarse mesh size and the overlap.

Finally, we will do some experiments to show the efficiency of our algorithm for problems with checkboard distributed coefficient, which equals 1 and $10^{i}(i=1,3,6)$ on adjacent subdomains. We assume that the coefficient is constant on each coarser element, and the coarser mesh size and the overlap size are fixed $(H=1 / 4, \delta=1 / 8)$. The 
TABLE 2: The iteration numbers with different mesh sizes $h$ and coefficient jumps $\left(10^{i}\right)$.

\begin{tabular}{cccccc}
\hline$i$ & \multicolumn{5}{c}{$h$} \\
& $1 / 8$ & $1 / 16$ & $1 / 32$ & $1 / 64$ & $1 / 128$ \\
\hline 1 & 14 & 15 & 15 & 16 & 16 \\
3 & 15 & 15 & 15 & 15 & 16 \\
6 & 15 & 15 & 15 & 15 & 15 \\
\hline
\end{tabular}

iteration numbers, with different mesh sizes $h$ and jumps in the coefficient, are reported in Table 2, from which, we conclude that our preconditioner also works well for problems with discontinuous coefficients at least in two dimensions.

\section{Competing Interests}

The authors declare that they have no competing interests.

\section{Acknowledgments}

This work was supported by NSFC under the Grants 11371199, $11301275,11371198,11526097$, and 11401294, the Opening Fund of Jiangsu Key Lab for NSLSCS under the Grant 201402, the Doctoral Fund of Ministry of Education of China under the Grant 20123207120001.

\section{References}

[1] J. Wang and X. Ye, "A weak Galerkin finite element method for second-order elliptic problems," Journal of Computational and Applied Mathematics, vol. 241, pp. 103-115, 2013.

[2] R. Zhang and Q. Zhai, "A weak Galerkin finite element scheme for the biharmonic equations by using polynomials of reduced order," Journal of Scientific Computing, vol. 64, no. 2, pp. 559$585,2015$.

[3] V. Ginting, G. Lin, and J. Liu, "On application of the weak Galerkin finite element method to a two-phase model for subsurface flow," Journal of Scientific Computing, vol. 66, no. 1, pp. 225-239, 2016.

[4] L. Chen, J. Wang, and X. Ye, "A posteriori error estimates for weak Galerkin finite element methods for second order elliptic problems," Journal of Scientific Computing, vol. 59, no. 2, pp. 496-511, 2014.

[5] B. Li and X. Xie, "A two-level algorithm for the weak Galerkin discretization of diffusion problems," Journal of Computational and Applied Mathematics, vol. 287, pp. 179-195, 2015.

[6] L. Chen, J. Wang, Y. Wang, and X. Ye, "An auxiliary space multigrid preconditioner for the weak Galerkin method," Computers \& Mathematics with Applications, vol. 70, no. 4, pp. 330-344, 2015.

[7] A. Toselli and O. Widlund, Domain Decomposition Methods Algorithms and Theory, Springer, New York, NY, USA, 2005.

[8] L. Wang and X. Xu, The Mathematical Basis of the Finite Element Method, Science Press, Beijing, China, 2005 (Chinese).

[9] S. Brenner and L. Scott, The Mathematical Theory of Finite Element Methods, Springer, New York, NY, USA, 2008. 


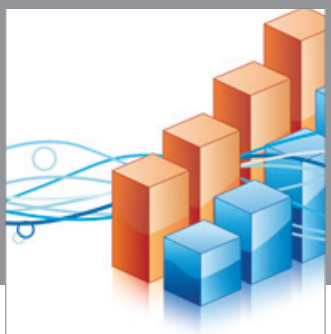

Advances in

Operations Research

vatem alat4

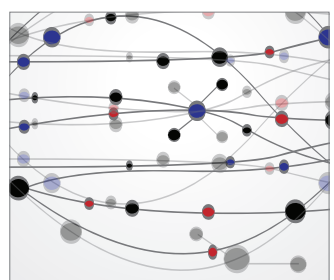

\section{The Scientific} World Journal
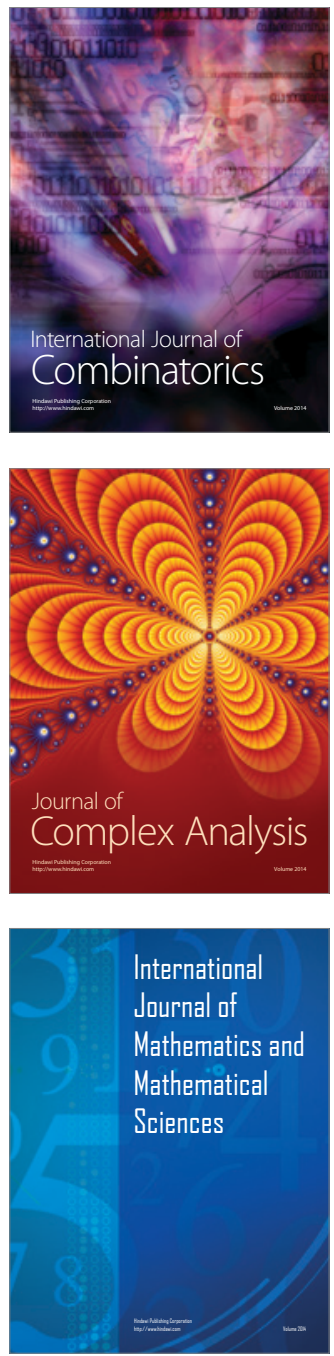
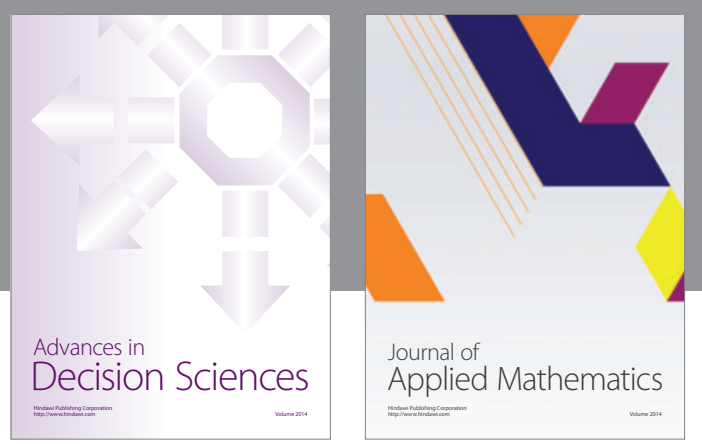

Algebra

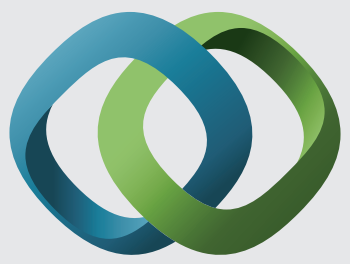

\section{Hindawi}

Submit your manuscripts at

http://www.hindawi.com
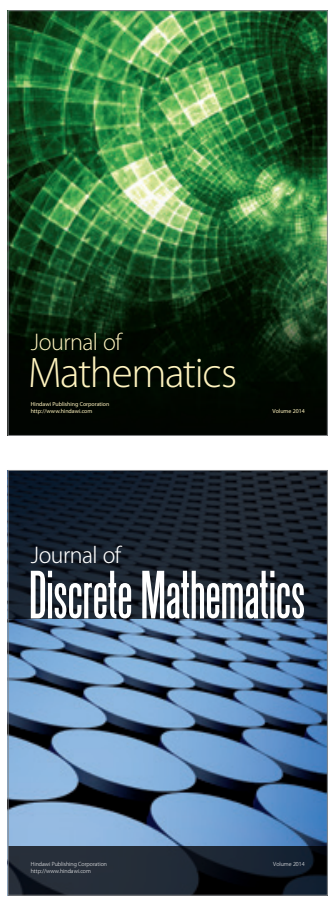

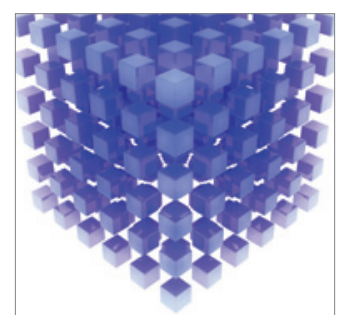

Mathematical Problems in Engineering
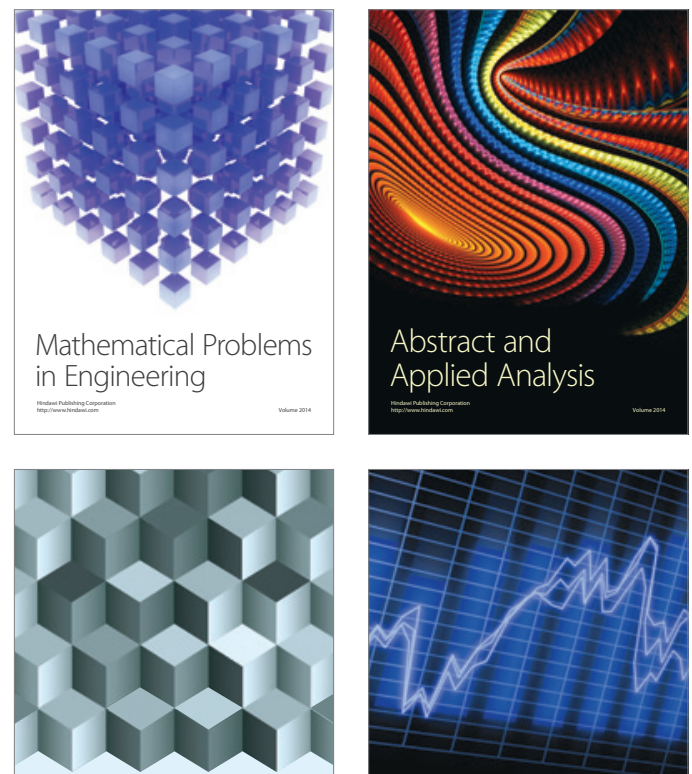

Journal of

Function Spaces

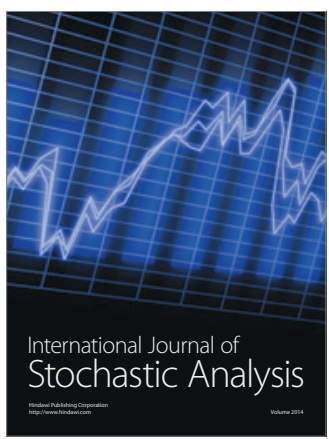

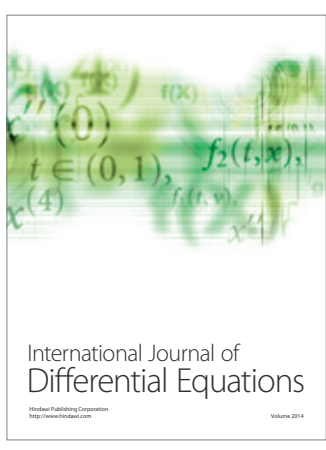
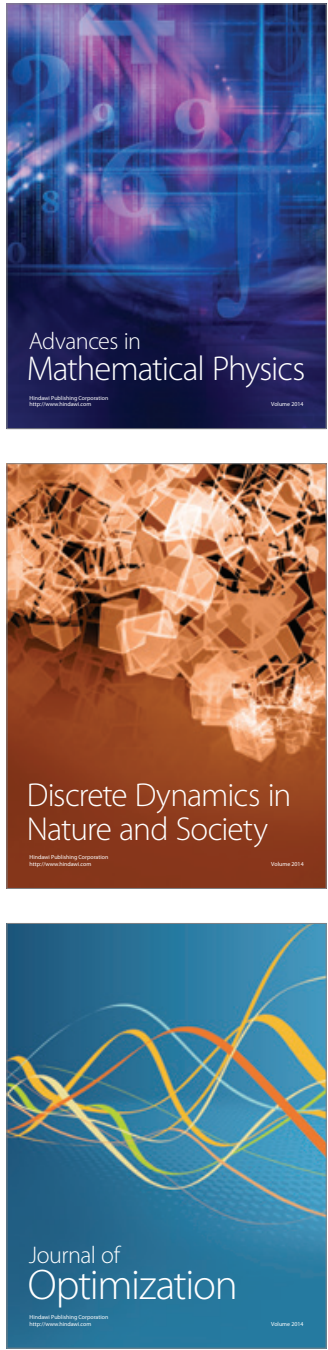